Cite this: Phys. Chem. Chem. Phys., 2014, 16, 2654

Received 20th September 2013 Accepted 12th December 2013

DOI: $10.1039 / c 3 c p 53994 f$

www.rsc.org/pccp

\section{Structural investigation of a hydrogen bond order-disorder transition in a polar one-dimensional confined ice $\dagger$}

\author{
Jasper Adamson, Nicholas P. Funnell, Amber L. Thompson and Andrew L. Goodwin* \\ The hydrogen-bond arrangement within crystalline 2,3,6,7,10,11-hexahydroxytriphenylene tetrahydrate \\ $\left(\mathrm{HHTP} .4 \mathrm{H}_{2} \mathrm{O}\right)$ undergoes an order-disorder transition at $240 \mathrm{~K}$, as evidenced by the emergence and \\ disappearance of systematic absence violations in variable-temperature single-crystal $\mathrm{X}$-ray diffraction \\ measurements. The low-temperature ordered phase is polar with ferroelectric coupling between \\ neighbouring one-dimensional ice-like columns of hydrogen-bonded $\mathrm{H}_{2} \mathrm{O}$ molecules. At temperatures \\ above $240 \mathrm{~K}$ the material adopts a paraelectric state characterised by the absence of long-range \\ ordering between column polarisations. We discuss the mapping of this phase transition onto the \\ problem of frustration on the canonical Ising square lattice, and suggest that $\mathrm{HHTP} \cdot 4 \mathrm{H}_{2} \mathrm{O}$ is an obvious \\ candidate for exploring switchable ferroelectric behaviour in confined ices.
}

Whether the dipolar nature of the water molecule might be translated into a polar form of ice is a question that has its origin in the very earliest studies of hydrogen bonding in solid phases of water. ${ }^{1,2}$ The configurational entropy of bulk ices is such that, to date, the only successful approaches to developing polar phases have involved symmetry lowering via e.g., surface layer deposition ${ }^{3,4}$ and dimensionality reduction in confined phases; ${ }^{5,6}$ even the nature of the supposedly-polar ice XI obtained on ion doping at low temperatures remains controversial. ${ }^{3,7,8}$ For some time, the focus of confinement studies has been on water-filled carbon and inorganic nanotubes. ${ }^{9-14}$ However, the primary application of polar ices-namely, in ferroelectric devices-relies on switchable bulk dipolar alignment, which is difficult for nanotube conglomerates; consequently the design strategy has recently shifted to exploiting aligned voids within nanoporous crystalline hosts. ${ }^{15,16}$ So, for example, water confined to nanochannels in the framework material $\mathrm{La}_{2} \mathrm{Cu}_{3}\left[\mathrm{NH}\left(\mathrm{CH}_{2} \mathrm{COO}\right)_{2}\right]_{6}$ exhibits antiferroelectric ordering below the confined liquid $\leftrightarrow$ solid transition at $350 \mathrm{~K} ;^{17}$ likewise, 1D "water wires" within a copper-based coordination polymer $\left[\mathrm{Cu}_{2}{ }^{\mathrm{I}} \mathrm{Cu}^{\mathrm{II}}(\mathrm{CDTA})\left(4,4^{\prime}\right.\right.$-bpy) $]$ have been shown to undergo a spontaneous transition between liquid and ferroelectric states at $277 \mathrm{~K}^{18}$

Department of Chemistry, University of Oxford, Inorganic Chemistry Laboratory, South Parks Road, Oxford OX1 3QR, UK. E-mail: andrew.goodwin@chem.ox.ac.uk; Fax: +44 (o)1865 274690; Tel: +44 (0)1865 272137

$\dagger$ Electronic supplementary information (ESI) available: Synthesis; experimental methods; X-ray single-crystal diffraction refinement details; order parameter calculations. CCDC 962532 and 962533. For ESI and crystallographic data in CIF or other electronic format see DOI: 10.1039/c3cp53994f
Despite the observation of dielectric hysteresis in the ferroelectric phase of $\left[\mathrm{Cu}_{2}{ }^{\mathrm{I}} \mathrm{Cu}^{\mathrm{II}}(\mathrm{CDTA})\left(4,4^{\prime}\right.\right.$-bpy $\left.)\right]$, perhaps the most obvious distinction between the formation of polar states in these confined ices and that in traditional ferroelectric phases such as $\mathrm{PbTiO}_{3}$ is that polarisation always seems to emerge on cooling from a liquid parent state rather than from a paraelectric phase. ${ }^{21}$ This suggests there exists only minimal distinction between the energy scales associated with long-range dipole alignment within and between nanopores and is likely the origin of the relatively weak and temperature-specific ferroelectric hysteresis observed for these systems. ${ }^{18}$

In this paper, we study the temperature-dependent evolution of 1D ice polarisation in the crystalline molecular hydrate HHTP. $4 \mathrm{H}_{2} \mathrm{O}$ [Fig. 1]. ${ }^{20}$ We show that $1 \mathrm{D}$ water-filled channels within HHTP. $4 \mathrm{H}_{2} \mathrm{O}$ exhibit a well-defined polar $\leftrightarrow$ paraelectric transition; as such the material is an excellent candidate for investigation into ice-based ferroelectric responses. HHTPcontaining systems are of intense current interest in the field of molecular framework materials, where they are favoured for their thermal robustness and attractive porosity/sorption properties. $^{22,23}$ Our focus is not on any covalent network assembled from HHTP, but rather on the hydrate of the molecule itself. As in all known HHTP cocrystals, the structure of this hydrate [shown in Fig. 1(b)] is dominated by the stacking of triphenylene cores into 1D columns; ${ }^{19,20,24}$ indeed this motif reflects the wellknown role of triphenylene as the structural basis for a large and separate family of discotic liquid crystals. ${ }^{25}$ The surface of these columns is decorated by hydrophilic - $\mathrm{OH}$ groups, and consequently the voids between columns are ideally suited to water coordination. In the single previous structural study of HHTP. 


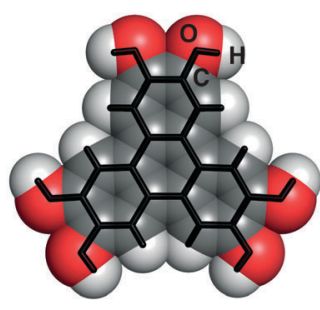

(a)

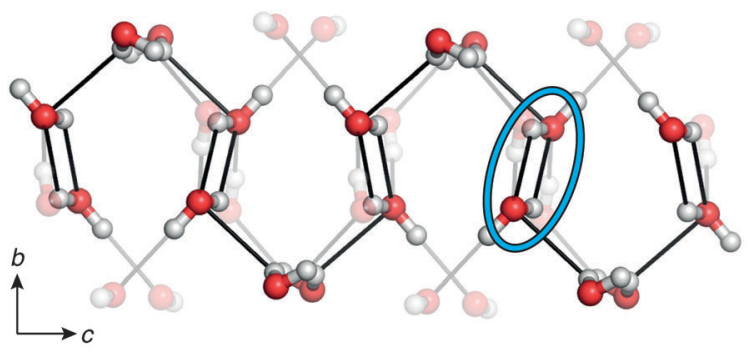

(c)

Fig. 1 (a) The HHTP molecule is planar with a hydrophobic core and hydrophilic exterior. (b) As in many solid triphenylenes, the crystal structure of HHTP. $4 \mathrm{H}_{2} \mathrm{O}$ consists of a triangular array of triphenylene columns. ${ }^{19,20}$ The voids between neighbouring HHTP columns are strongly hydrophilic. It is within these channels that the water molecules are located. (c) Neighbouring water molecules within a single pore are connected via hydrogen-bonding interactions to form a 1D ice with its chain axis parallel to the crystal $c$ axis. Some hydroxyl groups of the neighbouring HHTP molecules also participate in the hydrogen-bonding network; these are the transparent atoms and bonds shown here. In the reported structure of ref. 20 , the presence of inversion centres results in $\mathrm{O}-\mathrm{H} \ldots \mathrm{H}-\mathrm{O}$ contacts between some neighbouring $\mathrm{H}_{2} \mathrm{O}$ molecules (circled).

$4 \mathrm{H}_{2} \mathrm{O}$, it was shown that $\mathrm{H}_{2} \mathrm{O}$ molecules contained within these voids form an elaborate hydrogen-bonding network. ${ }^{20}$ Moreover, the water connectivity is one-dimensional such that the system can reasonably be considered a molecularly-confined $1 \mathrm{D}$ ice [Fig. 1(c)].

There were, however, two features of the ice-like hydrogenbonding network proposed that seemed to us to be counterintuitive and so prompted our own structural study. First, the crystal symmetry reported in ref. 20 was a centrosymmetric orthorhombic space group $P b c n$ that places inversion centres within the network itself. This crystallographic subtlety has the effect of forcing direct $\mathrm{O}-\mathrm{H} \cdot \cdots \mathrm{H}-\mathrm{O}$ contacts that would be considered improbable within the established framework of ice physics [Fig. 1(c)]. And, second, the anisotropic displacement parameters associated with the water $\mathrm{O}$ atoms were anomalously large considering the temperature at which data were collected (100 K; root-mean-squared displacement $\sqrt{U_{\max }} \simeq 0.4 \AA$ ).

In order to rationalise these crystallographic observations, we prepared a single-crystal sample of $\mathrm{HHTP} \cdot 4 \mathrm{H}_{2} \mathrm{O}$ and measured its X-ray diffraction pattern as a function of temperature (see ESI $\dagger$ for synthesis and experimental details) ${ }^{26-28}$ We collected diffraction data at $10 \mathrm{~K}$ intervals over the range $100-260 \mathrm{~K}$, throughout both heating and cooling regimes. For completeness, we note that prolonged exposure of the crystal to the nitrogen cryostream at temperatures above $260 \mathrm{~K}$ resulted in gradual desolvation, which explains our focus on temperatures below this value.
For all data sets, we were able to refine a satisfactory structural model based on the Pbcn solution of ref. 20; however, on close examination of the diffraction patterns themselves, we realised that a set of weak reflections forbidden in the $P b c n$ space group were unaccounted for by such a model. The intensity of these reflections was temperature dependent: they were strongest at the lowest temperatures but undetectable at $240 \mathrm{~K}$. Making use of the associated reflection conditions, we could assign to the lowtemperature structure the space group $P 2_{1} c n$, which is a maximal subgroup of $P b c n$ obtained by removing the inversion symmetry operation. Subsequent refinement of the $100 \mathrm{~K}$ data set in this lower-symmetry setting gave an improved structural model in which both the hydrogen-bonding connectivity and water $\mathrm{O}$ atom displacement parameters made physical sense. This model is illustrated in Fig. 2(a), and full structural details are given as ESI. $\dagger$

The distinction between $P 2_{1} c n$ and $P b c n$ structural models for low-temperature HHTP. $4 \mathrm{H}_{2} \mathrm{O}$ is crucial because the former is a polar space group whereas the latter is not. Indeed the structural feature responsible for polarity within the $P 2_{1} \mathrm{cn}$ model is precisely the ice-like hydrogen-bonding network

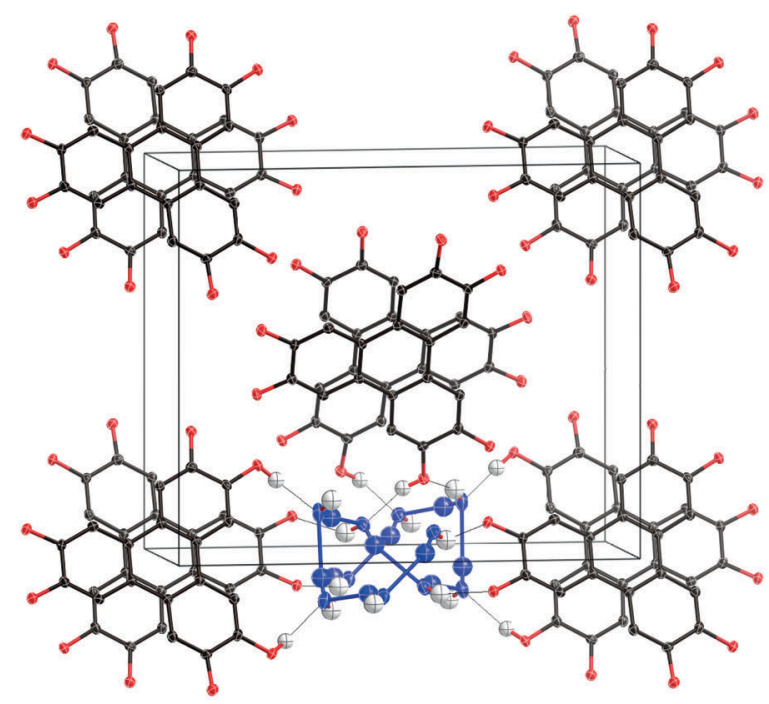

(a)

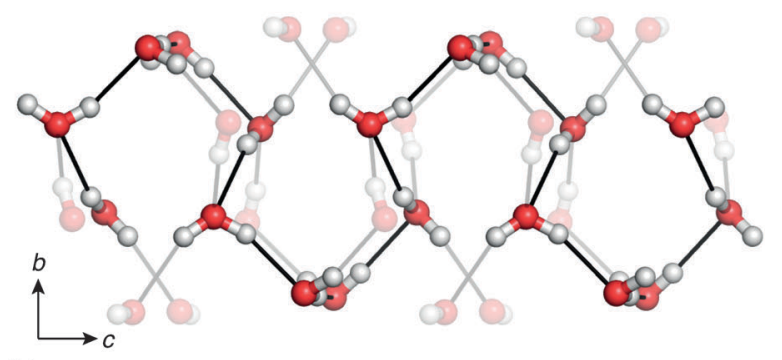

(b)

Fig. 2 (a) Representation of the $P 2_{1}$ cn structural model of lowtemperature HHTP. $4 \mathrm{H}_{2} \mathrm{O}$, refined against data collected at $100 \mathrm{~K}$. Most $\mathrm{H}$ and solvent atoms have been omitted for clarity. Shown in thermal ellipsoid representation is a single hydrogen-bonding network involving included water molecules. Thermal ellipsoids are drawn at the $50 \%$ probability level. (b) In the absence of inversion symmetry, the hydrogen-bonding arrangement within ice-like chains involves only $\mathrm{O}-\mathrm{H} \ldots \mathrm{O}$ contacts. 


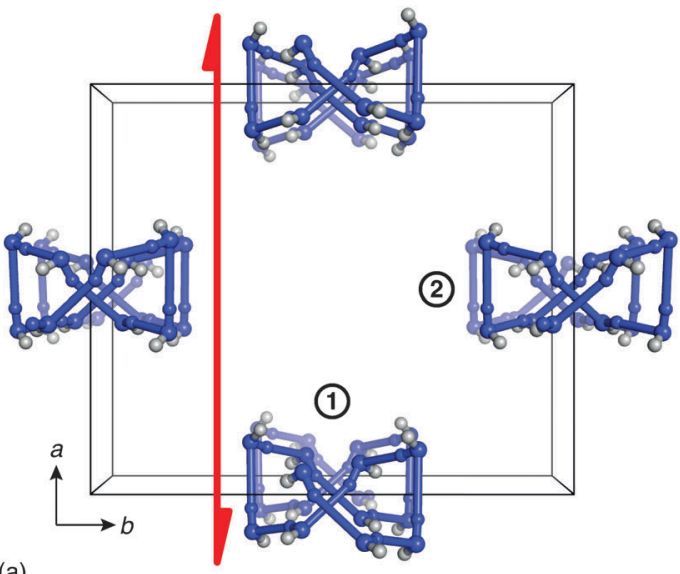

(a)

Fig. 3 (a) The low-temperature $P 2_{1} c n$ structure of HHTP. $4 \mathrm{H}_{2} \mathrm{O}$ contains two non-superimposable ice-like chains, here numbered ' 1 ' and ' 2 '. These are related by crystallographic symmetry; e.g. by the $2_{1}$ screw-axis shown. (b) These ice-like chains adopt directed meso-helical geometries, giving rise to a non-cancelling polarisation parallel to the a crystallographic axis. Arrows have been drawn on each meso-helix to emphasise the direction of $\mathrm{O}-\mathrm{H} \ldots \mathrm{O}$ hydrogen-bond donation. (c) Structure inversion has the effect of switching the direction of each $\mathrm{O}-\mathrm{H} \ldots$. O hydrogen bond, and hence reversing the polarisation along a. The left and centre panels illustrate the ice-like network for these two polarisation states. At temperatures above $240 \mathrm{~K}, \mathrm{HHTP} \cdot 4 \mathrm{H}_{2} \mathrm{O}$ exists in a paraelectric phase in which both states occur throughout the crystal structure, with no long-range correlation between neighbouring ice-like chains. The resulting average structure has $P b c n$ symmetry and is most meaningfully understood in terms of an equal-population superposition of the two polarisation states.

within the water-filled channels: i.e., the ice rules break inversion symmetry [Fig. 2(b)]. While there are two polar 1D ice chains within the crystallographic unit cell, these are related to one another by the crystal symmetry in a way that reinforces the polarisation component parallel to the $a$ crystal axis [Fig. 3]. In fact the chain geometry is of interest in its own right within the context of helical polymer motifs: ${ }^{29}$ it takes the rarely-observed form of a so-called "directed meso-helix", an object with polarisation components both along and normal to the helix axis. ${ }^{30}$ So whereas the polarisation associated with 1D ices is usually parallel to the confinement axis, ${ }^{16,18}$ in this system the net polarisation is perpendicular to that axis (the parallel component cancelling between adjacent chains). From a practical viewpoint, the advantage is that the crystal habit is to grow as square prisms with their long axis parallel to $c$ (i.e. the confinement axis-see ESI $\dagger$ ); consequently the surface area is maximised in perpendicular directions - the optimal geometry for exploiting any ferroelectric response.

Turning next to the X-ray diffraction data collected at $240 \mathrm{~K}$, we find that at this temperature the additional reflections forbidden in $\mathrm{Pbcn}$ but allowed in $P 2_{1} c n$ have zero intensity (within error). Consequently there is no crystallographic justification for lowering the symmetry beyond Pbcn in this case. As before, we obtain a numerically-satisfactory structure refinement using the model of ref. 20; however our earlier concerns regarding the physicality of this model would remain unaddressed. Instead we find that we obtain a better (and more meaningful) fit-to-data using a disordered Pbcn model containing equal proportions of the low-temperature $P 2_{1} c n$ structure and its inverse [Fig. 3(c)]. Such a model describes a paraelectric state in which the polarisations of neighbouring 1D hydrogen-bonding chains are sufficiently decoupled that long-range order disappears.

From a crystal chemical viewpoint, one might anticipate a relatively small enthalpy cost associated with interconversion of the two possible polarisation states. By comparing the HHTP molecule geometry in our $P 2_{1} c n$ structure with that observed in the $P b c n$ refinements (either ours for data collected at $240 \mathrm{~K}$, or that reported in ref. 20), we find a root-mean-squared deviation in atomic positions of less than $0.04 \AA$. Consequently the interconversion mechanism involves concerted proton shuttling within the ice-like chains, much as has been observed elsewhere for ferroelectric materials based on bistable hydrogen-bonding interactions. ${ }^{31,32}$ In the present case, the inter-coupling mechanism is via hydrogen bonding between ice-like chains and the hydroxyl groups of the HHTP molecules. Because the $\mathrm{H}_{2} \mathrm{O}-\mathrm{HHTP}$ hydrogenbonding topology is independent of chain polarisation, the degree of 'communication' between chains will be small indeed.

The transition from polar to paraelectric states of HHTP. $4 \mathrm{H}_{2} \mathrm{O}$ can be quantified using an order parameter derived from the intensities of those reflections unique to the $P 2{ }_{1} \mathrm{cn}$ space group (see ESI $\dagger$ ). Fig. 4 shows the temperature evolution of a small relevant portion of the diffraction pattern across the transition: here the three reflections (011) (031) and (071) are forbidden in $\mathrm{Pbcn}$ and can be seen to decrease in intensity with heating. For each of these reflections, the corresponding order parameter $\xi$ was calculated for each temperature according to

$$
\xi(0 k 1)=\alpha_{k}\left[\frac{I(0 k 1)}{I(042)}\right]^{1 / 2},
$$

where $\alpha_{k}$ is a temperature-independent scaling parameter, $I(h k l)$ is the measured intensity of the $(h k l)$ reflection, and the parent (042) reflection was used for normalisation for its combination of strong intensity at all temperatures and well-defined peak profile. The parameter $\xi$ takes the value of zero within the paraelectric regime and is non-zero whenever there is longrange correlation of the $1 \mathrm{D}$ ice polarisations. The temperature dependence of $\xi$ for these three reflections is illustrated in Fig. 4 , where the values at $240 \mathrm{~K}$ should be interpreted as the 


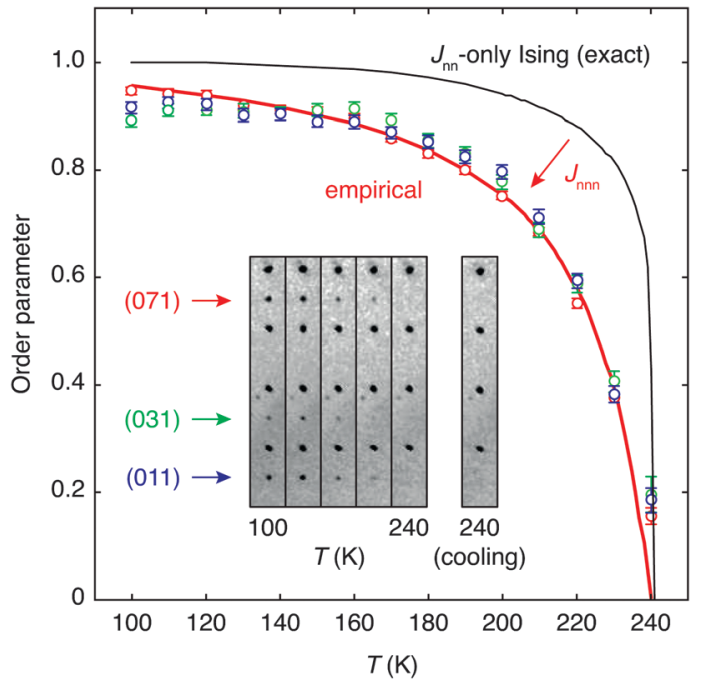

Fig. 4 Thermal variation in the order parameter $\xi$, calculated from the intensities of three representative systematic absence violations according to eqn (1) (data points). The absence of a clear discontinuity at the transition temperature $T_{\mathrm{C}} \simeq 240 \mathrm{~K}$ suggests the order-disorder transition is second order in nature. Relevant sections of the single-crystal diffraction patterns are shown in the inset; these illustrate the disappearance of $(0 k 1) ; k=2 n+1$ reflections on heating towards $T_{\mathrm{c}}$. The nearest-neighbour square lattice Ising model discussed in the text predicts a sharper transition (thin line) than is observed experimentally. ${ }^{33}$ The inclusion of frustrated next-nearest-neighbour terms in the ordering Hamiltonian is known to give rise to a temperature dependency similar to the empirical curve fit shown here [bold line; eqn (5)].

result obtained by integrating background fluctuations in the diffraction images. In the absence of a well-defined discontinuity in the function $\xi(T)$, we can conclude that the phase transition is likely to be second-order in nature. By comparing the results of heating and cooling runs, we find no evidence of hysteresis at the transition (see ESI $\dagger$ ); however the limiting magnitude of the order parameter $\xi(T \rightarrow 0)$ is only $\sim 75 \%$ the value obtained at the start of the experiment. This discrepancy may have one of two origins: either some desorption of $\mathrm{H}_{2} \mathrm{O}$ occurred on heating to $260 \mathrm{~K}$ or relaxation kinetics play a significant role in determining the degree of polar ordering over the experiment timescale.

To interpret the order parameter variation shown in Fig. 4 we construct a mapping of the polarisation state of $\mathrm{HHTP}_{4} 4 \mathrm{H}_{2} \mathrm{O}$ onto the square Ising model. Each 1D ice chain is capable of adopting either one of two configurations consistent with the ice rules-these are the orientations related to one another by the inversion centre present in $P b c n$ but absent in $P 2_{1} c n$ - and so can be assigned one of the two states $e \in\{1,-1\}$ allowed in an Ising model. For each 1D chain there are four equidistant nearest-neighbours to which its Ising state (i.e. polarisation) will be coupled via subtle reorganisation of the connecting HHTP molecules [Fig. 5(a)]. The corresponding energy scale $J_{\mathrm{nn}}$ is (by symmetry) identical for all nearest-neighbours of all chains, giving the canonical Hamiltonian

$$
\mathcal{H}=-J_{\mathrm{nn}} \sum_{i, j \in \mathrm{nn}} e_{i} e_{j}
$$

The topology of this coupled system is that of the square net and is two-dimensional because each $1 \mathrm{D}$ chain acts as a
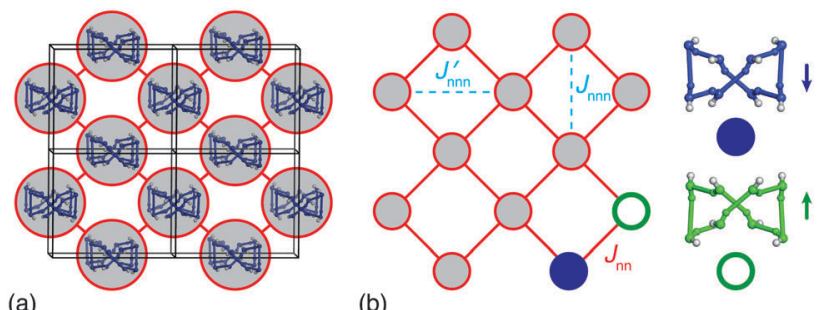

(a)

(b)

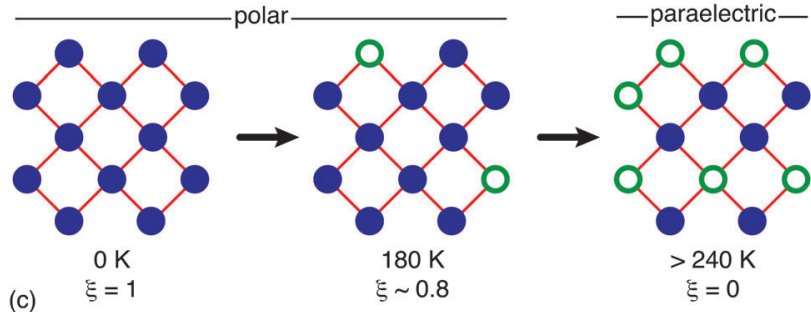

Fig. 5 (a) Each 1D ice-like chain interacts with four neighbouring chains via HHTP hydroxyl groups. (b) The network of coupled chains has the square lattice topology. Because each chain can adopt one of two polarisation states (indicated here by filled and open circles; cf. Fig. 3), there is a direct mapping between long-range ordering of ice polarisations in $\mathrm{HHTP} \cdot 4 \mathrm{H}_{2} \mathrm{O}$ and the general problem of ordering on the Ising square lattice. Here $J_{n n}$ describes the coupling strength between neighbouring 1D chains. By virtue of the orthorhombic crystal symmetry there are in fact two inequivalent next-nearest-neighbour couplings $J_{n n n}$ and $J_{n n n}$ ', the parity and relative magnitude of which will contribute to the discrepancy observed between experimental and theoretical $\xi(T)$ curves as shown in Fig. 4. (c) At temperatures below $T_{\mathrm{c}}$ the system adopts a polar state in which the majority of sites share the same state $(\xi>0)$, but at temperatures greater than $240 \mathrm{~K}$ there is an equal population of both states and the system is said to be paraelectric.

collective object. As such we can interpret the hydrogen bond order-disorder transition in HHTP. $4 \mathrm{H}_{2} \mathrm{O}$ as a specific manifestation of the general and well-established problem of the square Ising model [Fig. 5(b)]. ${ }^{33-35}$

In the case that nearest-neighbour interactions are the only interactions of relevance to the order-disorder process then the corresponding equilibrium behaviour of the square Ising model is known exactly. ${ }^{33}$ One anticipates a second-order phase transition between ordered (or, in the case of HHTP. $4 \mathrm{H}_{2} \mathrm{O}$, polar) and disordered (i.e., centrosymmetric) states at the critical temperature

$$
T_{\mathrm{c}}=\frac{J_{\mathrm{nn}}}{k_{\mathrm{B}}} \frac{2}{\ln (1+\sqrt{2})} .
$$

The temperature-dependence of the order parameter (三 magnetization in Onsager's formulation ${ }^{33}$ ) follows the closed form

$$
\xi=\left[1-\sinh ^{-4}\left(2 J_{\mathrm{nn}} / k_{\mathrm{B}} T\right)\right]^{1 / 8},
$$

which predicts a more sudden variation in $\xi(T)$ near $T_{\mathrm{c}}$ than we observe experimentally [Fig. 4].

In practice it is likely that next-nearest-neighbour interactions cannot altogether be neglected in a physical system such as we study here. The inclusion of appropriate additional terms into the Hamiltonian of eqn (2) leads to a surprisinglycomplex (and still controversial) system for which no exact 
solution is obtainable. ${ }^{35-37}$ Of relevance to the present situation is the observation that next-nearest-neighbour coupling constants $J_{\mathrm{nnn}}$ of opposite sign to $J_{\mathrm{nn}}$ have the effect of reducing the order parameter at lower temperatures to give a thermal variation more similar to that which we find to occur in HHTP. $4 \mathrm{H}_{2} \mathrm{O} .{ }^{35}$ This is the so-called 'frustrated Ising model', for which the phase transition is actually very weakly first-order. ${ }^{37}$ So there may be some suggestion that $1 \mathrm{D}$ ice polarisations couple in a ferroelectric sense for nearest neighbours in HHTP. $4 \mathrm{H}_{2} \mathrm{O}$ but in an antiferroelectric sense for next-nearest neighbours. Actually the situation is even more complex in this case because the orthorhombic crystal symmetry gives rise to two distinct types of next-nearest neighbours-a problem for which we are not aware of any prior theoretical groundwork. What is known is that the relationship between $J_{\mathrm{nn}}$ and $T_{\mathrm{c}}$ given in eqn (3) holds even for the frustrated Ising case, and so we can estimate the interchain coupling strength in HНTP. $4 \mathrm{H}_{2} \mathrm{O}$ to be $\sim 0.9 \mathrm{~kJ} \mathrm{~mol}^{-1}$. For comparison the interaction energy between two water molecules is estimated to lie between 18.8 and $30.1 \mathrm{~kJ} \mathrm{~mol}^{-1} ;^{38}$ consequently, this system displays an excellent discrimination between inter- and intra-chain energy scales. In the absence of a closed form for the temperature dependence of the order parameter for a frustrated square Ising model, we have used an empirical fit in Fig. 4 of the form

$$
\xi=\left(\frac{x}{1+x}\right)^{\nu},
$$

where $x=\alpha\left(T-T_{\mathrm{c}}\right) / T$, and $\alpha$ and $\nu$ are constants.

We attribute the accessibility of a bona fide paraelectric state in $\mathrm{HHTP} \cdot 4 \mathrm{H}_{2} \mathrm{O}$ to the very weak inter-chain coupling, which allows hydrogen bond disorder to occur without melting of the oxygen sublattice. We note specifically that the $\mathrm{H}_{2} \mathrm{O}$ oxygen atom positions remain well-defined even at temperatures above $T_{\mathrm{c}} \simeq 240 \mathrm{~K}$; the larger-than-expected oxygen displacement parameter observed reflects only the superposition of two half-occupied oxygen sites associated with each of the two polarisation states. This observation distinguishes the behaviour of paraelectric HHTP. $4 \mathrm{H}_{2} \mathrm{O}$ from that of the high-temperature phases of the related $1 \mathrm{D}$ ices $\left[\mathrm{Cu}\left(\mathrm{C}_{6} \mathrm{H}_{4} \mathrm{NO}_{2}\right)_{2}\right]$ and $\left[\mathrm{Cu}_{2}{ }^{\mathrm{I}} \mathrm{Cu}^{\mathrm{II}}(\mathrm{CDTA})\left(4,4^{\prime}\right.\right.$-bpy)$)$, within which long-range diffusive motion of the $\mathrm{H}_{2} \mathrm{O}$ occurs. Consistent with the small magnitude of $J_{\mathrm{nn}}$ is the negligible difference in HHTP geometries we observe across the order-disorder transition, which in turn explains the remarkable weakness of the additional diffraction intensities associated with symmetry breaking.

In conclusion, we find that the molecular hydrate НHTP. $4 \mathrm{H}_{2} \mathrm{O}$ exhibits a hydrogen bond order-disorder transition near $T_{\mathrm{c}} \simeq 240 \mathrm{~K}$ that is associated with the development at low temperatures of long-range ferroelectric coupling between polar 1D ice chains [Fig. 5(c)]. The phase transition behaviour we observe maps qualitatively onto the problem of ordering on the Ising square lattice, with the accessibility of a paraelectric phase at temperatures above $T_{\mathrm{c}}$ suggesting that ferroelectric switching of the low-temperature polar phase should be possible. Indeed we intend to pursue this line of investigation in the immediate future. Finally, we note that our study also illustrates how molecular systems might host confined ice phases in a manner normally associated with porous covalent networks or carbon/inorganic nanotube arrays.

The authors gratefully acknowledge financial support from the E.P.S.R.C. (EP/G004528/2) and the E.R.C. (Grant Ref.: 279705).

\section{References}

1 J. D. Bernal and R. H. Fowler, J. Chem. Phys., 1933, 1, 515-548.

2 S. T. Bramwell, Nature, 1999, 397, 212-213.

3 M. J. Iedama, M. J. Dresser, D. L. Doering, J. B. Rowland, W. P. Hess, A. A. Tsekouras and J. P. Cowin, J. Phys. Chem. B, 1998, 102, 9203-9214.

4 X. Su, L. Lianos, R. Shen and G. A. Somorjai, Phys. Rev. Lett., 1998, 80, 1533-1536.

5 C. F. Luo, W. Fa, J. Zhou, J. M. Dong and X. C. Zeng, Nano Lett., 2008, 8, 2607-2612.

6 F. Mikami, K. Matsuda, H. Kataura and Y. Maniwa, ACS Nano, 2009, 3, 1279-1287.

7 S. J. Singer, J.-L. Kuo, T. K. Hirsch, C. Knight, L. Ojamäe and M. L. Klein, Phys. Rev. Lett., 2005, 94, 135701.

8 S. J. Singer and C. Knight, in Advances in Chemical Physics, ed. S. A. Rice and A. R. Dinner, Wiley-Blackwell, Malden, U.S.A., 2012, vol. 147, pp. 1-74.

9 J. E. Bai, J. Wang and X. C. Zeng, Proc. Natl. Acad. Sci. U. S. A., 2006, 103, 19664-19667.

10 Y. Maniwa, H. Kataura, M. Abe, S. Suzuki, Y. Achiba, H. Kira and K. Matsuda, J. Phys. Soc. Jpn., 2002, 71, 2863-2866.

11 S. Ghosh, K. V. Ramanathan and A. K. Sood, Europhys. Lett., 2004, 65, 678-684.

12 Y. Maniwa, H. Kataura, M. Abe, A. Udaka, S. Suzuki, Y. Achiba, H. Kira, K. Matsuda, H. Kadowaki and Y. Okabe, Chem. Phys. Lett., 2005, 401, 534-538.

13 O. Byl, J.-C. Liu, Y. Wang, W.-L. Yim, J. K. Johnson and J. T. Yates, Jr., J. Am. Chem. Soc., 2006, 128, 12090-12097.

14 K. Koga, G. T. Gao, H. Tanaka and X. C. Zeng, Nature, 2001, 412, 802-805.

15 L. E. Cheruzel, M. S. Pometum, M. R. Cecil, M. S. Mashuta, R. J. Witterbort and R. M. Buchanan, Angew. Chem., Int. Ed., 2003, 42, 5452-5455.

16 B. Zhou, Y. Imai, A. Kobayashi, Z. Wang and H. Kobayashi, Chem. Lett., 2011, 1098-1100.

17 H. B. Cui, B. Zhou, L.-S. Long, Y. Okano, H. Kobayashi and A. Kobayashi, Angew. Chem., Int. Ed., 2008, 47, 3376-3380.

18 H.-X. Zhao, X.-J. Kong, H. Li, Y.-C. Jin, L.-S. Long, X. C. Zeng, R.-B. Huang and L.-S. Zheng, Proc. Natl. Acad. Sci. U. S. A., 2011, 108, 3481-3486.

19 T. L. Andresen, F. C. Krebs, N. Thorup and K. Bechgaard, Chem. Mater., 2000, 12, 2428-2433.

20 F. Thébault, L. Öhrström and M. Haukka, Acta Cryst., 2011, C67, 0143-0145.

21 R. J. Nelmes, R. O. Piltz, W. F. Kuhs, Z. Tun and R. Restori, Ferroelectrics, 1990, 108, 165-170.

22 C. J. Doonan, D. J. Tranchemontagne, T. G. Glover, J. R. Hunt and O. M. Yaghi, Nat. Chem., 2010, 2, 235-238. 
23 M. Hmadeh, Z. Lu, Z. Liu, F. Gándara, H. Furukawa, S. Wan, V. Augustyn, R. Chang, L. Liao, F. Zhou, E. Perre, V. Ozolins, K. Suenaga, X. Duan, B. Dunn, Y. Yamamto, O. Terasaki and O. M. Yaghi, Chem. Mater., 2012, 24, 3511-3513.

24 F. Toda, K. Tanaka, M. Takeshi, T. Nakai, I. Miyahara and K. Hirotsu, J. Phys. Org. Chem., 2000, 13, 39-45.

25 F. C. Krebs, N. C. Schiodt, W. Batsberg and K. Bechgaard, Synthesis, 1997, 1285-1290.

26 J. Cosier and A. M. Glazer, J. Appl. Crystallogr., 1986, 19, 105-107.

27 A. Altomare, G. Cascarano, C. Giacovazzo, A. Guagliardi, M. C. Burla, G. Polidori and M. Camalli, J. Appl. Crystallogr., 1994, 27, 435.

28 P. W. Betteridge, J. R. Carruthers, R. I. Cooper, K. Prout and D. J. Watkin, J. Appl. Crystallogr., 2003, 36, 1487.

29 L. Han and M. Hong, Inorg. Chem. Commun., 2005, 8, 406-419.
30 L. Plasseraud, H. Maid, F. Hampel and R. W. Saalfrank, Chem.-Eur. J., 2001, 7, 4007-4011.

31 Y.-Y. Yin, X.-Y. Chen, X.-C. Cao, W. Shi and P. Cheng, Chem. Commun., 2012, 48, 705-707.

32 M. Szafranński, A. Katrusiak and G. J. McIntyre, Phys. Rev. Lett., 2002, 89, 215507.

33 L. Onsager, Phys. Rev., 1944, 65, 117-149.

34 C. N. Yang, Phys. Rev., 1952, 85, 808-816.

35 D. P. Landau, Phys. Rev. B, 1980, 21, 1285-1297.

36 A. Kalz, A. Honecker and M. Moliner, Phys. Rev. B, 2011, 84, 174407.

37 S. Jin, A. Sen, W. Guo and A. W. Sandvik, Phys. Rev. B, 2013, 84, 144406.

38 H. Yu and W. F. van Gunsteren, J. Chem. Phys., 2004, 121, 9549-9564. 